\title{
Evaluation and optimal design of supersonic nozzle for laser-assisted oxygen cutting of thick steel sections
}

Article in International Journal of Advanced Manufacturing Technology • January 2016

DOI: 10.1007/s00170-015-8268-7

CITATIONS

0

4 authors, including:

\section{Chi Zhang}

Tsinghua University

2 PUBLICATIONS 0 CITATIONS

SEE PROFILE

\section{Xuejun Fan}

Chinese Academy of Sciences

53 PUBLICATIONS 407 CITATIONS

SEE PROFILE
READS

71

\section{Yueming Yuan}

Chinese Academy of Sciences

10 PUBLICATIONS 6 CITATIONS

SEE PROFILE 


\title{
Evaluation and optimal design of supersonic nozzle for laser-assisted oxygen cutting of thick steel sections
}

\author{
Chi Zhang $^{2} \cdot$ Peng Wen ${ }^{1,2} \cdot \underline{\text { Yueming Yuan }}^{3} \cdot \underline{\text { Xuejun Fan }}^{3}$
}

Received: 17 July 2015 / Accepted: 17 December 2015

(C) Springer-Verlag London 2016

\begin{abstract}
With the full use of combustion heat from Fe-O reaction, laser-assisted oxygen cutting (LASOX) is able to cut thick mild steel plates by a defocused laser of medium power (approximately $1 \mathrm{~kW}$ ). The dynamic behavior of oxygen gas flow, determined by nozzle structure and cutting parameters, is the key factor affecting cut ability and quality. It is significant to understand the method to design a suitable supersonic nozzle and to evaluate its performance. A supersonic minimum length nozzle (MLN) was designed and made specially for LASOX. The configuration $\left(D_{\text {exit }} / D_{\text {critical }}\right)$ of the designed nozzle was chosen based on two-dimensional isentropic calculation of dynamic gas pressure. The divergent section of the MLN was designed in accordance with the method of characteristics (MOC). Numerical simulation and Schlieren visualization were used to observe and compare the flow behavior of the free jet from the designed MLN nozzle and a commercial supersonic nozzle. The MLN nozzle generates a highly uniform and stable supersonic jet extending a longer distance along the axis of nozzle, which is around 1.4 times longer than that from the commercial supersonic nozzle. Mild steel plates in $20 \mathrm{~mm}$ thickness were cut to investigate the effect of cutting parameters on LASOX cut quality of two nozzles. The MLN significantly improved cutting quality
\end{abstract}

Peng Wen

wenpeng@tsinghua.edu.cn

1 State Key Laboratory of Tribology, Tsinghua University, Beijing 100084, China

2 Department of Mechanical Engineering, Tsinghua University, Beijing 100084, China

3 State Key Laboratory of High Temperature Gas Dynamics, Institute of Mechanics, Chinese Academy of Sciences, Beijing 100190, China due to the better performance of gas flow. The optimal cut quality is related to both oxygen pressure and cutting speed. The oxygen content was measured along the cut surface to explain the difference in cut quality.

Keywords Laser cutting $\cdot$ Supersonic nozzle $\cdot$ Numerical simulation $\cdot$ Flow visualization

\section{Introduction}

Laser cutting is widely used in sheet metal industry. The prominent advantages include fast speed, good quality, high flexibility, etc. One of the biggest challenges lies in how to cut thick sections regarding the further expanding application, especially in heavy industry like shipbuilding, chemical, and nuclear engineering. The usual cutting thickness of mild steel is no more than $20 \mathrm{~mm}$ for industrial applications of laser cutting. The limitation is mainly resulted by two aspects: the energy input and the melt ejection. Higher energy input can be solved by higher power laser source, as well as the use of oxygen as the assist gas providing exothermic energy from the Fe-O reaction. The melt ejection can be enhanced by supersonic gas flow and wider cut kerf. For example, 20-mm mild steel sections can be cut by laser oxygen cutting usually with power more than $4 \mathrm{~kW}$ and kerf width about $0.5-0.7 \mathrm{~mm}$ $[1,2]$. However, even with further increased laser power, the cut thickness is difficult to increase further, let alone the high cost of higher laser power source. The key problem is the melt ejection. The gas flow inside the cut kerf easily becomes turbulent, which deteriorates the melt ejection especially for a high ratio of thickness/width regarding cutting thick sections $[3,4]$. Meanwhile, the instability of the oxidation process influenced by the oxygen flow behavior can also cause the deterioration of cut ability and cut quality [5-7]. Thus, it is 
significant to study the behavior of gas flow in order to improve cut thickness for laser cutting with lower cost and higher stability.

Laser-assisted oxygen cutting (LASOX), developed by O'Neil, makes it possible to use a medium laser power to cut thick steel plates [8]. A 1-kW defocused laser spot, whose diameter is larger than that of the nozzle exit, heats up the surface of cut pieces and initiates Fe-O combustion reaction. Basically, the oxidation process and the melt ejection are dominated by supersonic oxygen gas flow. This method makes full use of the continuous and sufficient exothermic heat from the $\mathrm{Fe}-\mathrm{O}$ reaction, thus significantly releases the heat input requirement from laser power. Besides, the melt ejection is also improved by enlarging the kerf width to the size close to the nozzle exit diameter. The reported cutting thickness has increased to $50 \mathrm{~mm}$. However, a supersonic nozzle has to be used to ensure a uniform and stable supersonic jet extending the whole cut thickness at least. LASOX is regarded more sensitive to nozzle structure and cutting parameters compared with conventional laser cutting methods. Sundar [9] developed a regression analysis model to describe the effect of the independent processing parameters on cut quality for LASOX. Artificial neural network was also used to optimize cutting parameters for LASOX [10]. It was found that gas pressure and cutting speed had pronounced effect on cut quality. However, nozzle type and structure are not investigated as a key factor in the above papers.

As is known, subsonic and transonic nozzles are not suitable for cutting thick plates because of the energy loss and dynamic fluctuations caused by the shock waves when the inlet gas pressure is high. Supersonic nozzles have been commonly used for cutting thick plates in oxy-fuel flame cutting due to the stable and uniform jet even at high pressures. The application of supersonic nozzles is increasingly adopted in laser cutting of thick plates as well. The early researches on supersonic nozzles for laser cutting can refer to Man and Duan's work $[11,12]$. They pointed out the design principles to obtain a uniform jet for a supersonic nozzle with inert gas. The inlet pressure $P_{0}$ should be equal to the design value $P_{\mathrm{d}}$, which corresponds to the configuration $\left(D_{\text {exit }} / D_{\text {critical }}\right)$ and the Mach number of the exit jet. However, they did not further study the selection principle of $P_{\mathrm{d}}$ (configuration and exit Mach number) to improve the melt ejection in laser cutting. Besides, the high-pressure oxygen flow plays a more important role both in the melt ejection and the exothermic heat.

Some reports have been found on the design method of a suitable supersonic nozzle for LASOX. Ermolaev et al. [13] firstly studied the effect of nozzle structure on the cut velocity and quality. Through three-dimensional simulations of a model kerf, it was found that the gas dynamics inside a wide cut kerf is more like a free jet into the atmosphere. If the narrow cut kerf, whose width is much smaller than the nozzle exit diameter, it is easy to cause flow separation and deflection. Thus, the three-dimensional gas dynamics inside the cut kerf can be simplified to a two-dimensional free jet approximately during LASOX cutting. It was also found that nozzles with throat diameter less than $1 \mathrm{~mm}$ are not suitable for LASOX due to friction effect. However, the influence of exit Mach number $\left(P_{\mathrm{d}}\right.$ and $\left.D_{\text {exit }} / D_{\text {critical }}\right)$ and the profile of the divergent section were not taken into consideration. For a commercial supersonic nozzle, the divergent section is always so long that it easily interferes with the defocused laser inside the nozzle and causes thick boundary layers inside the nozzle for LASOX. Besides, it is significant how to choose the optimal design pressure (namely, the exit Mach number and $\left.D_{\text {exit }} / D_{\text {critical }}\right)$, which is relevant to the gas momentum to blow off molten slag as well as to the status of $\mathrm{Fe}-\mathrm{O}$ reaction. The relationship between nozzle structure and the optimal cutting parameters seems still unclear for LASOX. This work is to propose a design method for a suitable supersonic nozzle to cut thick steel sections. The flow field characteristics of the designed nozzle were compared with those of a commercial supersonic nozzle both by numerical simulation and flow visualization. Cutting trials are done to show the effect of nozzle structure and cutting parameters on cut quality.

\section{Optimal design of supersonic nozzle}

\subsection{Configuration design}

As Fig. 1 shows, a supersonic nozzle consists of four parts: stable, convergent, throat, and divergent sections. The stable section makes the inlet flow uniform and non-turbulent. It is better to have a big diameter and a long length in this section if permitted. The convergent section is to accelerate gas flow, as well as to keep the flow uniform. Generally speaking, this section is easy to meet the need only if the profile is smooth, continuous, and convergent. Here, it is set to be straight. The inlet diameter $D_{0}$ is $10 \mathrm{~mm}$ and the length $L_{2}$ is $19.6 \mathrm{~mm}$ for the convergent section.

When the inlet pressure $P_{0}$ equals to the design pressure $P_{\mathrm{d}}$, the exit velocity of the jet from the supersonic nozzle reaches the design Mach number $M_{\mathrm{d}}$. Therefore, for a certain supersonic nozzle, the dynamic characteristics of the free jet are related to the value of $P_{\mathrm{d}}$ and $M_{\mathrm{d}}$. The two values are determined by $D_{\text {exit }} /$ $D_{\text {critical }}$, respectively indicating the diameter of the exit and the throat of the nozzle. Based on the isentropic gas dynamic theory, friction shear force and impact pressure on the cut front, which are the two main 
Fig. 1 Schematic of supersonic nozzle

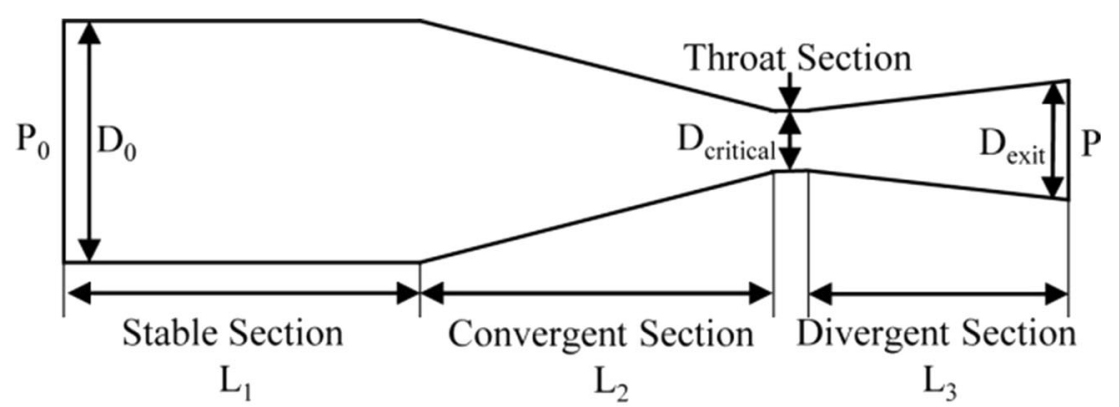

driving force to blow off the molten slag, can be calculated by (1) and (2) [14].

$$
\begin{aligned}
\tau_{f} & =0.03 \operatorname{Re}^{-\frac{1}{5}} \rho u^{2} \approx 3 \times 10^{-3} \rho u^{2} \\
\rho u^{2} & =\rho c^{2} M^{2}=\rho \gamma R T M^{2} \\
& =\gamma R M^{2} \rho_{0} T_{0} \cdot \frac{\rho}{\rho_{0}} \cdot \frac{T}{T_{0}} \\
& =\gamma P_{0} M^{2} \cdot \frac{1}{\left(1+\frac{\gamma-1}{2} M^{2}\right)^{\frac{1}{\gamma-1}}} \cdot \frac{1}{1+\frac{\gamma-1}{2} M^{2}} \\
& =P_{0} \cdot \gamma M^{2}\left(1+\frac{\gamma-1}{2} M^{2}\right)^{-\frac{\gamma}{\gamma-1}} \\
\rho u^{2} & =P_{0} \cdot f(M)
\end{aligned}
$$

$\tau_{\mathrm{f}}$ is the friction force; Re is the Reynold number; $P_{0}, T_{0}$, and $\rho_{0}$ are the total pressure, temperature, and density of the jet at the inlet. $\rho, u, T$, and $M$ are the density, velocity, temperature, and Mach numbers of the jet at the exit; $\gamma$ is the ratio of specific heat and equals to 1.4 when the jet is pure oxygen. It can be seen that both friction force $\tau_{\mathrm{f}}$ and impact pressure depend on $\rho u^{2}$, which can be further calculated by Eq. (3).

Equation (3) shows that $\rho u^{2}$ depends on the inlet pressure $P_{0}$ and a function of the exit Mach number $f(M)$. The relationship between the function $f(M)$ and $M$ is shown in Fig. 2. When $M$ increases, $f(M)$ increases at first, peaks when $M=1.414$, and then decreases. However, it should be noted that $f(M)$ is more precise theoretically when $M$ is chosen as $M_{\mathrm{d}}$

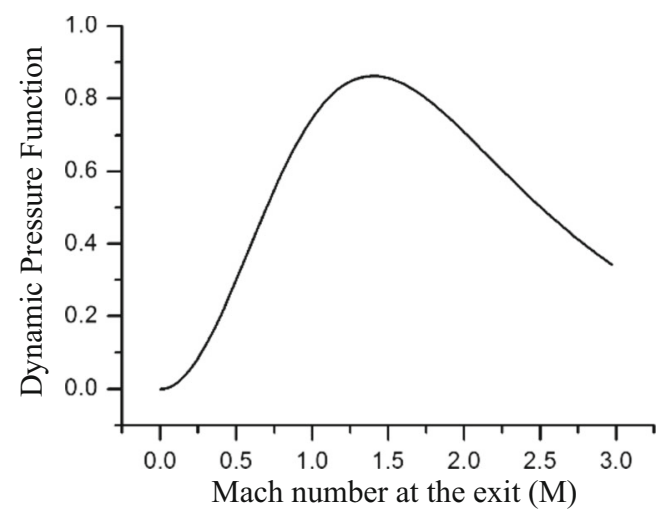

Fig. 2 Relationship between the dynamic function and the exit Mach number considering isentropic transformation. Namely, the inlet pressure $P_{0}$ should be equal or close to the design pressure $P_{\mathrm{d}}$ in order to avoid shocks and fluctuations in gas jet. Therefore, the suitable oxygen pressure range should be determined first before choosing an $M_{\mathrm{d}}$ for an optimal supersonic nozzle. The value of $f(M)$ should be high on condition that the corresponding $P_{0}$ is within the determined pressure range.

The common pressure range used in LASOX is about 0.6$1.0 \mathrm{MPa}$, so $P_{\mathrm{d}}$ is chosen as $0.8 \mathrm{MPa}$ here. The corresponding $M_{\mathrm{d}}$ is 2.0. The configuration parameter $D_{\text {exit }} / D_{\text {critical }}$ is set as 1.32 by isentropic calculation. The diameter of oxygen gas jet is usually kept about $2 \mathrm{~mm}$ in order to control kerf width to benefit the melt ejection as well as oxygen consumption. If the diameter is too small, there is not enough space for the melt ejection; on the contrary, the oxygen consumption is so high that it brings in high cost and too much molten slag to blow off. The diameter of the defocused laser spot should be bigger than that of the oxygen jet and is usually about $2 \sim 3 \mathrm{~mm}$. For such a big defocused spot, the focal distance of the laser lens must be short and the throat diameter $D_{\text {critical }}$ of the nozzle must be big enough to avoid the possible heat damage to nozzles due to direct laser radiation on the throat. Here, the used focal distance is $123 \mathrm{~mm}$, and the used laser spot is $3.5 \mathrm{~mm}$. $D_{\text {critical }}$ is $2.0 \mathrm{~mm}$, and the exit diameter $D_{\text {exit }}$ is $2.64 \mathrm{~mm}$ therefore.

\subsection{Profile design}

After $D_{\text {exit }}$ and $D_{\text {critical }}$ of a supersonic nozzle are chosen, the profile of the divergent section is the key to obtain a uniform and stable supersonic jet. Several methods can be used to calculate the profile of the divergent section, such as semi-analytical, empirical formula and method of characteristics (MOC) method. Assuming that the jet flow is non-viscous and does not form a boundary layer, MOC can obtain a divergent profile for shock-free with a minimum length. The minimum length of the divergent section is very helpful to avoid the interference between the defocused laser and the inner chamber of the nozzle in LASOX. Therefore, the designed nozzle by MOC is named as the MLN. 
The performance of each point in the gas flow is obtained by solution to the point on the characteristic lines. Equation (4) shows the dynamic equation of a two-dimension steady irrotational axisymmetric gas flow. Equation (5) defines the two characteristic lines through each point in the flow field. Equation (6) defines the relationship of two velocity components $u$ and $v$ along each Mach line.

$\left(u^{2}-a^{2}\right) u_{x}+2 u v u_{y}+\left(v^{2}-a^{2}\right) v_{y}-\frac{a^{2} v}{y}=0$

$\left(\frac{d y}{d x}\right)_{ \pm}=\lambda_{ \pm}=\tan (\theta \pm \mu)$

$\left(u^{2}-a^{2}\right) d u_{ \pm}+\left[2 u v-\left(u^{2}-a^{2}\right) \lambda_{ \pm}-\frac{a^{2} v}{y}\right] d x_{ \pm}=0$

Where $a=a(u, v)$ is the sound velocity; $\lambda$ is the gradient of the characteristic line; $\theta$ is the streamline angle; $\mu$ is the Mach angle; the subscript + and - means left-running and rightrunning, respectively.

In order to solve $u$ and $v$ of each point in the flow field, grids have to be drawn to make two characteristic lines intersect. The solution process is shown in Fig. 3a-e schematically. The computation process starts at the initial line with the initial expansion condition at the throat radius. Once two points are solved, a third point can be solved by two new characteristic lines from the two points, as shown in Fig. 3a, b. More points can be solved either by two known points or by one point plus its symmetric point, as shown in Fig. 3c, d. The profile of the divergent section can be solved by numerical integration according to mass conservation law on the characteristic lines leaded from the solved boundary characteristic line. The intersection point to the characteristic lines is obtained with the Euler-predictor scheme, while the corrector scheme iterates the predicted property and updates the intersection point until the desired convergence criterion is obtained.

The profile of the divergent section for the MLN with a sharp corner was calculated by using software MATLAB ${ }^{\circledR}$, as shown in Fig. 4. The length of divergent section $L_{3}$ is $3.11 \mathrm{~mm}$. The used number of characteristic lines was set to be 50 according to the balance between computational accuracy and manufacturing ability. More characteristic lines mean more accuracy, which needs more precise manufacturing ability. The MLN was manufactured by a high-precision lathe to make sure that the inner chamber is smooth enough. Meanwhile, a commercial supersonic nozzle is used to make comparison. The divergent curve of the used commercial supersonic nozzle is straight with a divergent angle of $5^{\circ}$. The working pressure of the commercial supersonic nozzle is $0.7-$ $0.8 \mathrm{MPa}$.
Fig. 3 Schematic of MOC solution process

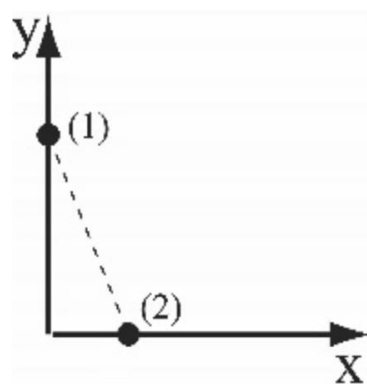

(a)

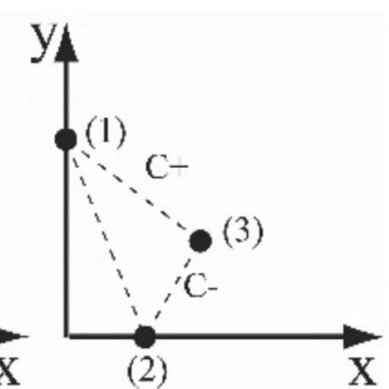

(b)



(c)

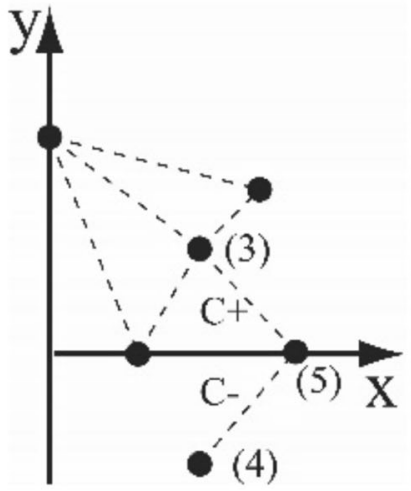

(d)

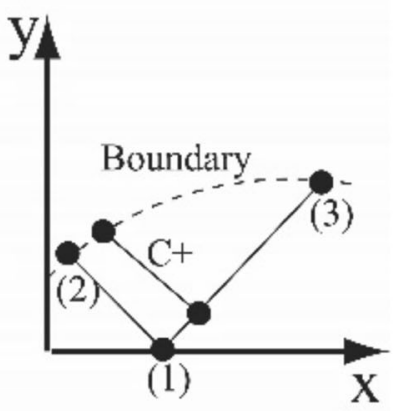

(e) 


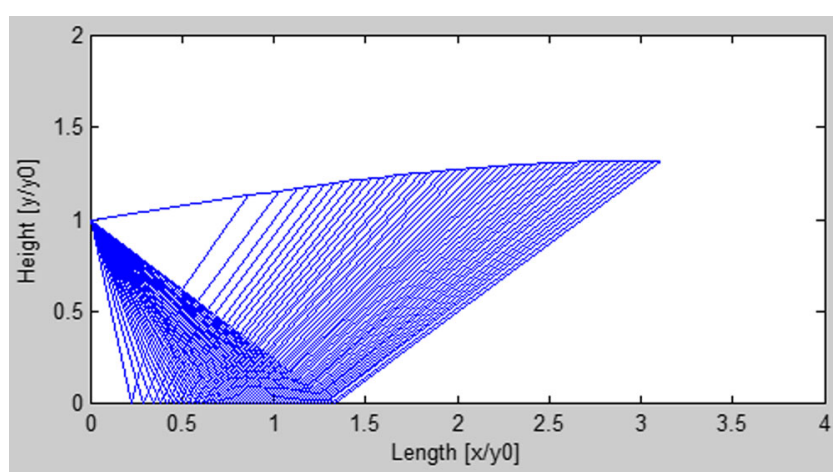

Fig. 4 Profile of the divergent section of the designed MLN

\section{Performance evaluation of supersonic nozzle}

\subsection{Numerical simulation}

Commercial software FLUENT ${ }^{\circledR}$ was used to simulate twodimensional axisymmetric free jet. The oxygen flow is assumed as the ideal gas, while the viscosity obeys the Sutherland law. The computational area is set as $40 \mathrm{~mm} \times 40 \mathrm{~mm}$ to model the entire cutting region. The grids are much fine as $0.05 \mathrm{~mm}$ near the axis to capture the details of shock structure. Figure 5 gives the calculated flow velocity of the MLN and the commercial supersonic nozzle on the working condition when the total inlet pressure is $0.8 \mathrm{MPa}$. It can be seen that the jet from the MLN is more uniform and stable along the centerline. Few shock cells and velocity fluctuations can be seen downstream the jet. The jet from the commercial supersonic nozzle is not as uniform as that from the MLN. Shock cells and fluctuations of velocity can be seen along the centerline. The supersonic length of the jet from the MLN nozzle is about $40 \mathrm{~mm}$, which is $8 \mathrm{~mm}$ longer than that from the commercial supersonic nozzle.

Figure 6 shows the calculation results of axial velocity and dynamic pressure from the MLN and the commercial supersonic nozzle when the inlet pressure is $0.8 \mathrm{MPa}$. Small deviations of velocity and dynamic pressure at the nozzle exit can be seen due to different boundary layer growth in the divergent section. The uniform and stable length of velocity and dynamic pressure from the MLN is around $32 \mathrm{~mm}$, which is 1.5 times longer than that of the commercial supersonic nozzle. After that, both axial velocity and dynamic pressure drop down dramatically. Both longer stable length and smaller fluctuations theoretically indicate that the MLN is more suitable for cutting thick sections.

\subsection{Flow visualization}

A Z-type Schlieren optical system with a tungsten halogen lamp was used to visualize the free jet from the MLN and the commercial supersonic nozzle. The experimental setup is shown in Fig. 7. The disturbance of gas flow under the cutting nozzle provokes a change in gas pressure and causes a change of the refractive index, which deviates the collimated light beam through the test area. The non-deviated light beam is filtered by a geometrical knife edge. The deviated light beam is projected onto the image plane. A digital camera captures the dynamic behavior of gas flow under the cutting nozzle. The diameter of the main mirror is $250 \mathrm{~mm}$. The power of the halogen lamp is $250 \mathrm{~W}$.

Figure 8 shows Schlieren images of the free jet flow of the two nozzles under different total inlet pressures. The total inlet pressure equals to the gauge pressure plus the ambient pressure. The "bright-dark" changes in the images indicate compression-expansion waves and fluctuations of gas properties. The core region of the jet, where viscous effect is negligible, allows a uniform velocity and pressure. A conical region is indicated by the change in refractive index along the boundaries of the supersonic region. The change in refractive index allows an approximation of the supersonic length measured as $L_{\mathrm{s}}$. For the commercial supersonic nozzle, $L_{\mathrm{s}}$ increases and the fluctuation of the jet becomes stronger by increasing the inlet pressure. The strong fluctuation is thought to be caused by an improper expansion in the divergent section. The under-expansion can be found at the nozzle exit. The
Fig. 5 Simulation result of velocity contour from the commercial supersonic and MLN. Distance from axis and nozzle exit (mm). Comparison of the axial dynamic pressure

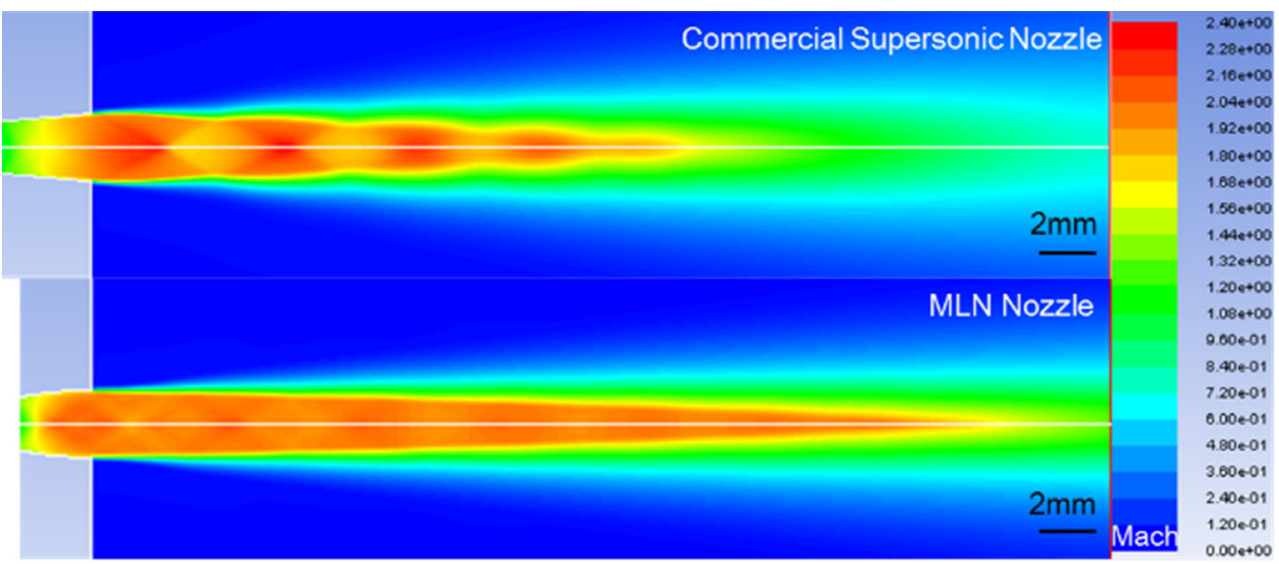




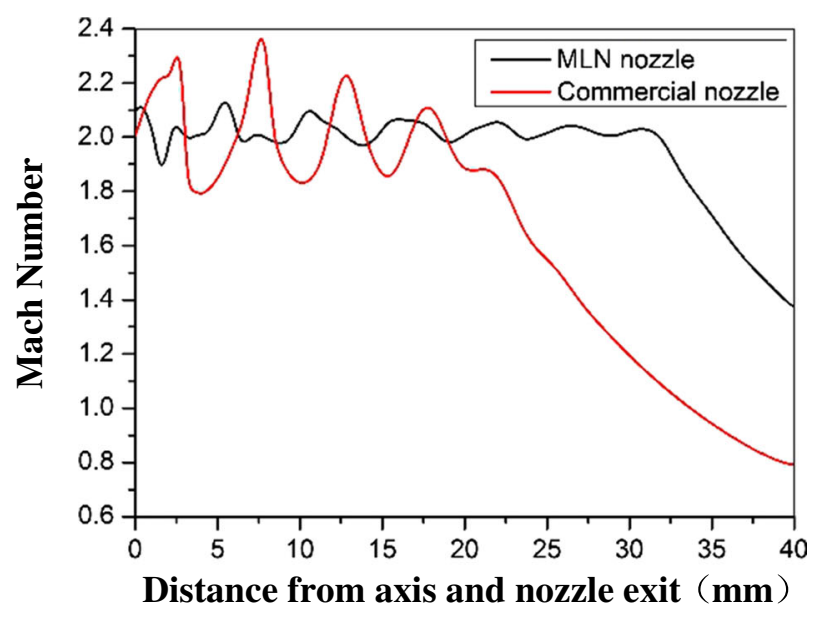

(a) Comparison of the axial velocity

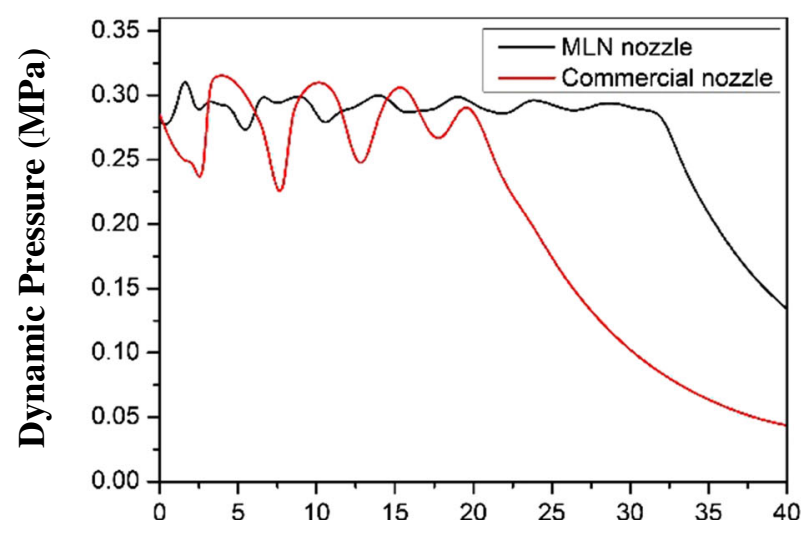

Distance from axis and nozzle exit ( $\mathbf{m m}$ )

(b) Comparison of the axial dynamic pressure

Fig. 6 Comparison of velocity and dynamic pressure along the nozzle axis between the MLN and the commercial supersonic nozzle

jet continues to expand not only axially but also radially judged by the wavy shape of the jet. The expansion waves go to the jet boundary and are reflected as compression waves. The compression waves intersect and form normal shocks

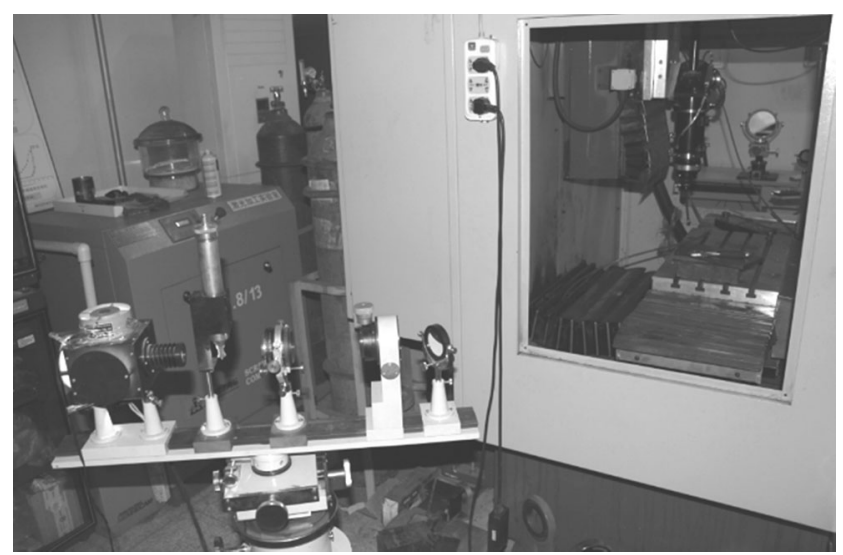

Fig. 7 Experimental setup of Schlieren method shown as the "dark" region in Fig. 8a, which considerably deteriorates the gas properties and decreases the supersonic length $L_{\mathrm{s}}$. Therefore, the commercial supersonic nozzle used is not proper for thick section cutting.

For the MLN in Fig. 8b, it can be seen that when the inlet pressure increases, the supersonic length $L_{\mathrm{s}}$ increases as well. When the inlet pressure is as high as $0.7 \mathrm{MPa}$, the fluctuation is still quite weak. Little "bright-dark" changes are seen in the jet. It means that the jet expands properly in the divergent section and does not under-expand at the nozzle exit. Axial and radial expansion does not happen in such situation, so the shape of the jet is straight with no wavy structure. There is no "dark" region found in the jet with inlet pressure $0.7 \mathrm{MPa}$, which is approximate to the designed pressure $0.8 \mathrm{MPa}$. It means that few shocks form in the jet with the MLN. The effective supersonic length of the jet from the MLN is about $36 \mathrm{~mm}$ when the total inlet pressure is $0.7 \mathrm{MPa}$, which is 1.4 times longer than that from the commercial supersonic nozzle. The flow visualization results coincide well with the numerical results. Therefore, the design of the divergent section of the MLN nozzle is proper and can help to improve cut quality.

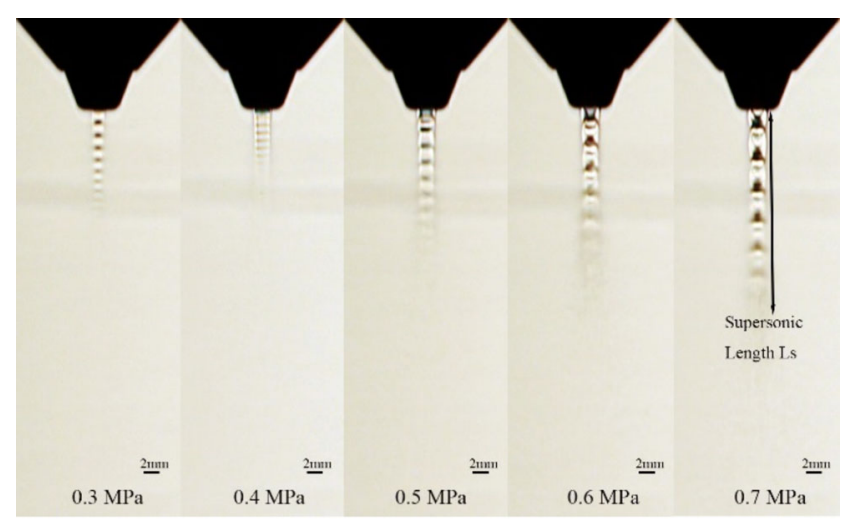

(a) Commercial supersonic nozzle

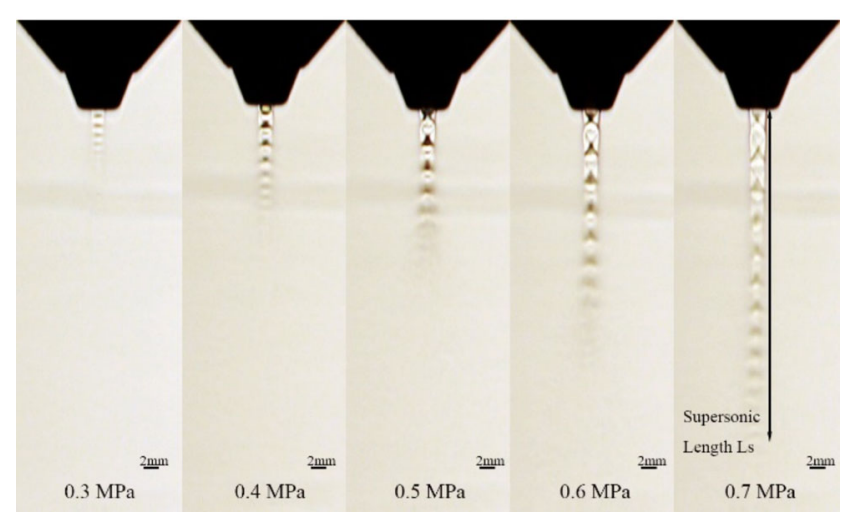

(b) MLN nozzle

Fig. 8 Schlieren visualization under different inlet pressures 


\subsection{Cutting trials}

An IPG 2-kW fiber laser was used as power source. The feeding fiber and processing fiber were, respectively, 50 and $100 \mu \mathrm{m}$. The focal distance was $123 \mathrm{~mm}$ for the used focus lens. The diameter of laser spot was set to be $3.5 \mathrm{~mm}$. Laser power was kept to be $1 \mathrm{~kW}$. Q235 mild steel plates with $150 \times 60 \times 20 \mathrm{~mm}$ (length, width, and thickness) were used as cut pieces. The MLN and commercial supersonic nozzle described above were used to carry out cutting trials. After cutting trials, the oxygen content along the cut surface was measured by energy dispersive X-ray detector (EDX).

The key cutting parameters include stand-off distance, oxygen pressure, and cutting speed. Stand-off distance describes the distance between the nozzle tip and the surface of workpiece. A short stand-off distance makes full use of the effective gas flow and is good for cut quality but easy to burn the nozzle tip. It was fixed as $1 \mathrm{~mm}$ in cutting trials. For supersonic nozzles, the optimal inlet pressure should be equal to the designed value in theory. In a practical condition, there is an allowable range for the operating pressure rather than a fixed value however. The optimal operating pressure is close to the designed value, and the specific value is related to other cutting parameters, especially cutting speed. Figures 9 and 10 respectively show the cutting surface with different oxygen pressure for the commercial nozzle and MLN. The cutting direction is from right to left.

From Fig. 9, unstable burning can be seen at the beginning and at the end of the cut surface. The pressure here means the total inlet pressure. When the inlet pressure is $0.6 \mathrm{MPa}$, dross attachment is severe at the bottom. It means that the supersonic gas flow deteriorates at the lower part of the cut surface and makes the dross difficult to blow off. When the inlet pressure is $0.7 \mathrm{MPa}$, dross attachment is less severe. Deep gouging can be seen at the bottom of the cut surface though. With increasing gas pressure, the gas properties at the lower part become better due to increased momentum. However, higher inlet pressure brings greater radial expansion as well and causes side burning and over-reaction. It is also seen that the striation pattern is different at the upper and lower parts of the cut surface. Striation at the upper part is fine and intensive while coarse and scattered at the lower part. The optimal pressure range for the commercial supersonic nozzle is pretty narrow. When the inlet pressure is $0.65 \mathrm{MPa}$, gouging and dross attachment can still be seen but less severe compared to lower and higher pressures.

From Fig. 10, it can be seen that the cut quality is all acceptable using the MLN when the inlet pressure varies from 0.6 to $0.7 \mathrm{MPa}$. The cut surface cut is smooth and straight. The striation pattern is fine, intensive, and shallow in the whole cut surface. Little gouging and dross attachment are seen at the bottom. The simulation and flow visualization results indicate that the supersonic jet from the MLN is longer and more

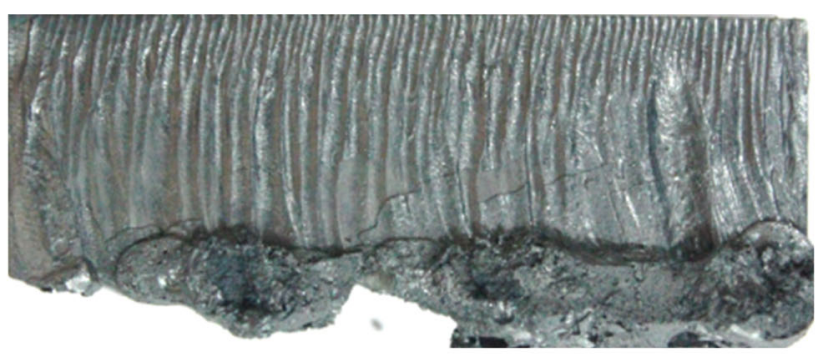

(a) Pressure 0.6MPa Velocity $0.15 \mathrm{~m} / \mathrm{min}$ STD $1 \mathrm{~mm}$

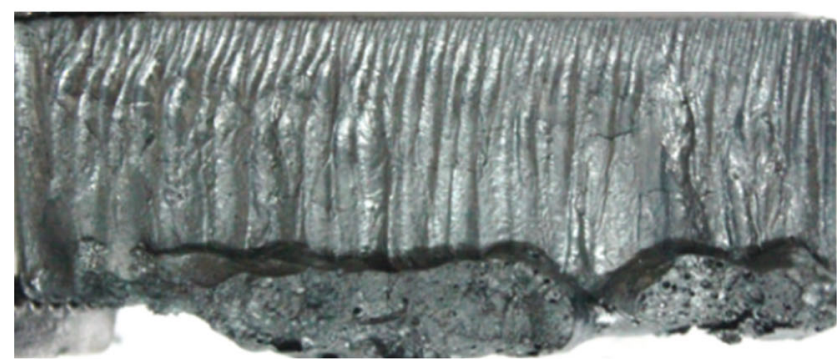

(b) Pressure 0.65MPa Velocity $0.15 \mathrm{~m} / \mathrm{min}$ STD $1 \mathrm{~mm}$

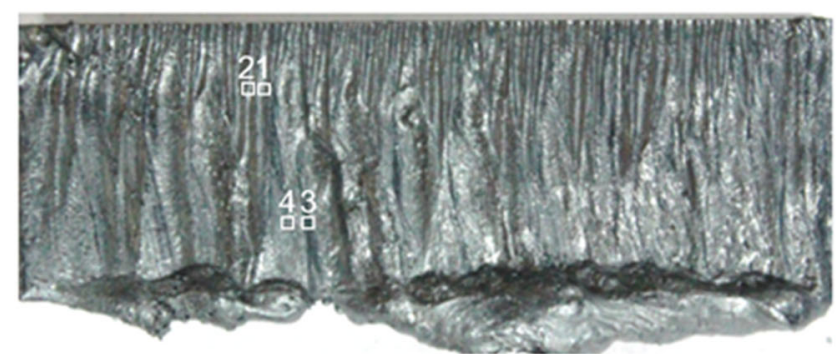

(c) Pressure 0.7MPa Velocity $0.15 \mathrm{~m} / \mathrm{min}$ STD $1 \mathrm{~mm}$

Fig. 9 Influence of oxygen pressure on cut quality with the commercial supersonic nozzle

uniform and stable. Higher impact pressure and shear force can be obtained, which can blow off molten slag efficiently. The radial expansion and fluctuations of flow properties is less as well for the MLN. It keeps the side burning effect to the minimum and shortens the residence time of the molten slag, leaving little dross at the bottom of the cut surface. Higher flow velocity at the lower part of the cut front can also make the impurity boundary layer thinner, which benefits to the oxidation process to generate more energy transferring downstream the section. Therefore, the optimal pressure range with the MLN is wider than that of the commercial supersonic nozzle, and the cut quality is improved.

From Figs. 10 and 11, it can be seen that the striations on the cut surface is straight and parallel when the cutting velocity is $0.2 \mathrm{~m} / \mathrm{min}$. When increasing cutting velocity to $0.25 \mathrm{~m} / \mathrm{min}$, the striation at the lower part of the surface starts to slant against the cutting direction. The inclined angle is increased when increasing cutting velocity further to $0.3 \mathrm{~m} / \mathrm{min}$. The position where the striation begins to slant goes up with increasing cutting velocity, as the dash line shows in Fig. 11. More dross 


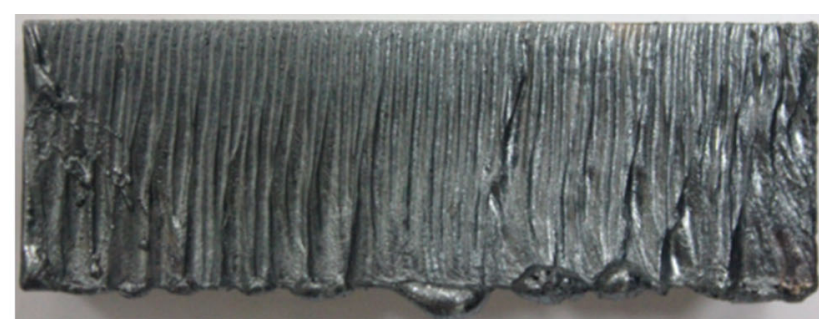

(a) Pressure 0.6MPa Velocity $0.20 \mathrm{~m} / \mathrm{min}$ STD $1 \mathrm{~mm}$

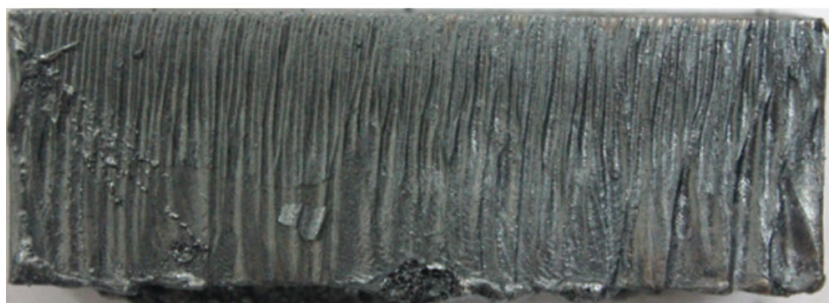

(b) Pressure 0.65MPa Velocity $0.20 \mathrm{~m} / \mathrm{min}$ STD $1 \mathrm{~mm}$

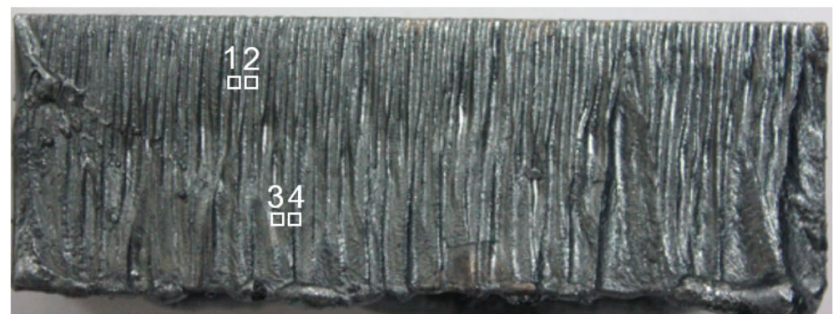

(c) Pressure 0.7MPa Velocity $0.20 \mathrm{~m} / \mathrm{min}$ STD $1 \mathrm{~mm}$

Fig. 10 Influence of gas pressure on cut quality of 20-mm mild steel cutting with designed MLN

attachment at the bottom of the cut surface can be seen when the cutting velocity increases. Lose-cut

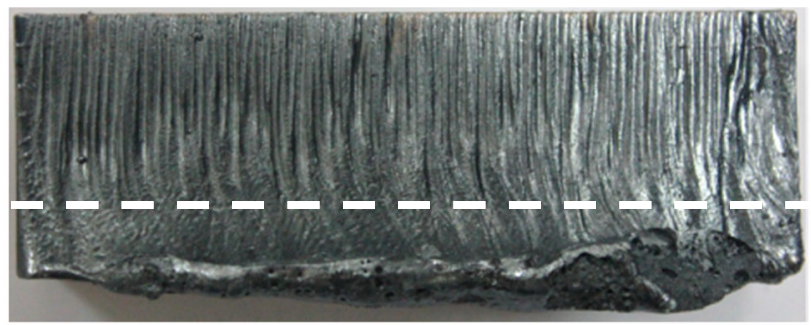

(a) Pressure 0.7MPa Velocity $0.25 \mathrm{~m} / \mathrm{min}$ STD $1 \mathrm{~mm}$

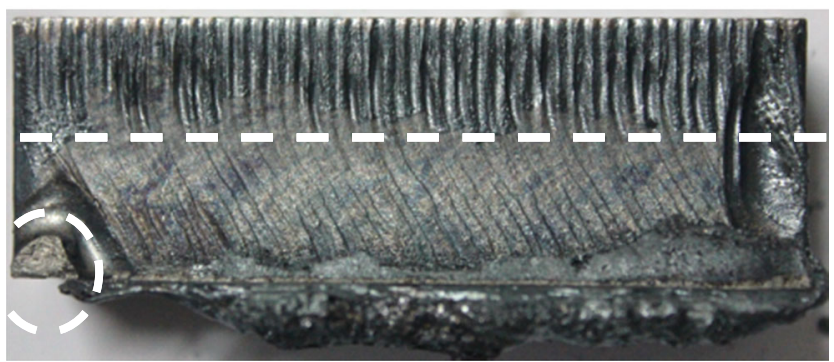

(b) Pressure 0.7MPa Velocity $0.30 \mathrm{~m} / \mathrm{min}$ STD $1 \mathrm{~mm}$

Fig. 11 Influence of cutting velocity on cutting ability and quality phenomenon can be seen at the bottom of cut surface, as the dash circle shows in Fig. 11b.

The striation becomes finer and closer in the upper part of the cut surface with increasing cutting velocity. However, inclination of the striations, dross and lose cuts become the limitations for high velocity. These deteriorations are regarded due to the deterioration of gas dynamics at the lower part of the cutting surface. It is studied that flow separation effect is not as severe when the kerf width is close to the nozzle exit diameter and the cut front is coaxial with the jet boundary [11]. However, when the cutting velocity exceeds the optimal range, the incline angle of the cut front becomes much bigger and the displacement distance between the nozzle axis and cut front becomes smaller even coaxial. The insufficient oxidation process at the upper part causes narrow cut kerf at the upper part. These factors make flow separation happen during practical LASOX process $[15,16]$. Below the separation point, streamlines detach from the cut front, so the impact pressure and friction force exerted on the cut front is much weaker. The molten metal gathers at the lower part of the kerf, so the melt ejection becomes difficult, causing poor cut quality. Further study needs to be done to investigate the cut front geometry and flow separation on LASOX in order to improve cut ability and quality further.

In LASOX, it is basically the combustion reaction of $\mathrm{Fe}$ and $\mathrm{O}$ that occurs in the cut kerf, instead of melting metal. The reaction product is made up of iron oxides or Fe-O mixtures. The oxygen flow not only burns metal but also blows off the molten Fe-O mixtures. Thus, the oxygen content can indicate much information about the cut quality. Table 1 shows the oxygen content along the cut surface obtained by the designed MLN and the commercial supersonic nozzle. The measured positions can be found schematically in Figs. 9c and 10c. Points 1 and 2 are located in the upper side, while points 3 and 4 are in the lower side. For the MLN, the oxygen content is lower in the upper side than that in the lower side. For the commercial supersonic nozzle, the result is just opposite. Moreover, the oxygen content in upper side for the MLN cut surface is lower than that made by the commercial supersonic nozzle, while it is higher in the lower side.

The thickness and oxygen content of residual oxide layer along the cut surface are decided by the dynamic behavior of

Table 1 Oxygen content along cut surface (wt $\%)$

\begin{tabular}{lll}
\hline & MLN nozzle & Commercial supersonic nozzle \\
\hline Point 1 & 12.9 & 20.2 \\
Point 2 & 11.4 & 18.2 \\
Average 1 & 12.1 & 19.2 \\
Point 3 & 19.6 & 8.9 \\
Point 4 & 19.7 & 9.6 \\
Average2 & 19.7 & 9.3 \\
\hline
\end{tabular}


oxygen flow. Usually, the thickness is small in the upper side and big in the lower side along the cut surface for a proper cut. The oxygen content in the upper side is lower than that in lower side as well. This is the case for the results of the MLN. However, for the commercial supersonic nozzle, things are different because the cut quality was poor and a lot of dross remained at the bottom.

From numerical simulation (Fig. 6) and Schlieren visualization (Fig. 8), it can be seen that the fluctuation of velocity and dynamic pressure of the commercial supersonic nozzle is much bigger than that of the MLN near the nozzle exit. The fluctuation deteriorates the melt ejection in the upper side. Thus, the oxygen content is high in the upper side along the cut surface for the commercial supersonic nozzle. For the lower side along the cut surface, the gas flow from the commercial supersonic nozzle becomes so turbulent and weak that the ambient air entrains into the oxygen flow. It decreases the purity of oxygen flow. The Fe-O reaction continues when the oxygen diffuses across liquid $\mathrm{FeO}$ layer to the interface of fresh iron. The purity of oxygen is key to maintain sufficient combustion. Thus, the oxygen content is much lower than that obtained by the MLN. The low oxygen content increases the viscosity of the Fe-O mixture and is harmful to the melt ejection. It partly explains the much remained dross at the bottom of the cut surface for the commercial supersonic nozzle.

\section{Conclusions}

A supersonic nozzle for LASOX was designed. Numerical simulation, flow visualization, and cutting trials were done to investigate the nozzle structure and cutting parameters on cutting performance.

1. An MLN nozzle with a shorter divergent section was designed and manufactured. The configuration $\left(D_{\text {exit }} /\right.$ $\left.D_{\text {critical }}\right)$ of the nozzle was chosen according to twodimensional isentropic gas dynamic theory. The profile of the divergent section was determined by method of characteristics.

2. Numerical simulation and flow visualization showed that the MLN generated a highly uniform and stable cutting oxygen jet with a relatively high dynamic pressure at the nozzle exit. Compared to the used commercial supersonic nozzle, the length of supersonic flow produced by the MLN was longer and the gas behavior inside the supersonic flow was with less fluctuation.

3. Cutting trials were done with 20-mm-thick mild steel sections by LASOX method. Compared to the commercial supersonic nozzle, cut ability and quality were improved significantly by using the MLN. Cutting velocity was the key factor on cut quality. Little dross, straight, and fine striations were observed on the cut surface with optimal cutting velocity. When the cutting velocity exceeded the optimal range, inclined striations, dross, and lose cuts appeared on the lower part of the cut surface, indicating a deteriorated cut quality.

Acknowledgments This work was supported by the Tsinghua University Initiative Scientific Research Program.

\section{References}

1. Malikov AGE, Orishich AM, Shulyat'ev VB (2009) Experimental optimization of the gas-assisted laser cutting of thick steel sheets. Quantum Electron 39(6):547

2. Schulz W, Hertzler C (2004) Cutting: modeling and data in laser applications. Springer, Berlin Heidelberg, pp 187-218

3. Kovalev OB, Yudin PV, Zaitsev AV (2009) Modeling of flow separation of assist gas as applied to laser cutting of thick sheet metal. Appl Math Model 33(9):3730-3745

4. Schulz W, Nießen M, Eppelt U, Kowalick K (2009) Simulation of laser cutting. In The theory of laser materials processing. Springer Netherlands: 21-69

5. Ivarson A, Powell J, Magnusson C (1996) The role of oxygen pressure in laser cutting mild steels. J Laser Appl 8(4):191-196

6. Yao YL, Chen H, Zhang W (2005) Time scale effects in laser material removal: a review. Int J Adv Manuf Technol 26(5-6): 598-608

7. Chen K, Yao YL, Modi V (1999) Numerical simulation of oxidation effects in the laser cutting process. Int J Adv Manuf Technol 15(11):835-842

8. O’Neill W, Gabzdyl JT (2000) New developments in laser-assisted oxygen cutting. Opt Lasers Eng 34(4):355-367

9. Sundar M, Nath AK, Bandyopadhyay DK, Chaudhuri SP, Dey PK, Misra D (2009) Effect of process parameters on the cutting quality in lasox cutting of mild steel. Int J Adv Manuf Technol 40(9-10): 865-874

10. Chaki S, Ghosal S (2011) Application of an optimized SA-ANN hybrid model for parametric modeling and optimization of LASOX cutting of mild steel. Prod Eng 5(3):251-262

11. Man HC, Duan J, Yue TM (1998) Dynamic characteristics of gas jets from subsonic and supersonic nozzles for high pressure gas laser cutting. Opt Laser Technol 30(8):497-509

12. Man HC, Duan J, Yue TM (1997) Design and characteristic analysis of supersonic nozzles for high gas pressure laser cutting. J Mater Process Technol 63(1):217-222

13. Ermolaev GV, Kovalev OB, Zaitsev AV (2013) Parameterization of hybrid laser-assisted oxygen cutting of thick steel plates. Opt Laser Technol 47:95-101

14. White FM, Corfield I (2006) Viscous fluid flow, vol Vol.3. McGraw-Hill, New York

15. Wandera C, Kujanpaa V (2010) Characterization of the melt removal rate in laser cutting of thick-section stainless steel. J Laser Appl 22(2):62-70

16. Brandt AD, Settles GS (1997) Effect of nozzle orientation on the gas dynamics of inert-gas laser cutting of mild steel. J Laser Appl 9(6):269-277 